\title{
Neutralization Characteristics of HIV-1 CRF01_AE Env Clones From Infections in China
}

\author{
Xinyu Zhang \\ National Institutes for Food and Drug Control (NIFDC) and WHO Collaborating Center for Standardization and Evaluation of \\ Biologicals
}

Zehua Zhou

National Institutes for Food and Drug Control (NIFDC) and WHO Collaborating Center for Standardization and Evaluation of Biologicals

Xueli Li

National Institutes for Food and Drug Control (NIFDC) and WHO Collaborating Center for Standardization and Evaluation of Biologicals

Yimeng An

National Institutes for Food and Drug Control (NIFDC) and WHO Collaborating Center for Standardization and Evaluation of Biologicals

\section{Fei Jiang}

National Institutes for Food and Drug Control (NIFDC) and WHO Collaborating Center for Standardization and Evaluation of Biologicals

\section{Youchun Wang}

National Institutes for Food and Drug Control (NIFDC) and WHO Collaborating Center for Standardization and Evaluation of Biologicals

\section{Feng Gao}

Jilin University

\section{Weijin Huang}

National Institutes for Food and Drug Control (NIFDC) and WHO Collaborating Center for Standardization and Evaluation of Biologicals

\section{Lingjie Liao}

National Center for AIDS/STD Control and Prevention, Chinese Center for Disease Control and Prevention

\section{Hui Xing}

National Center for AIDS/STD Control and Prevention, Chinese Center for Disease Control and Prevention

\section{Bin Yu}

Jilin University

\section{Chenyan Zhao ( $\nabla$ zcy1210@163.com )}

National Institutes for Food and Drug Control (NIFDC) and WHO Collaborating Center for Standardization and Evaluation of Biologicals

\section{Research Article}

Keywords: HIV-1, CRF01_AE, neutralizing antibody, pseudovirus

Posted Date: September 22nd, 2021

DOI: https://doi.org/10.21203/rs.3.rs-568729/v2

License: (a) (1) This work is licensed under a Creative Commons Attribution 4.0 International License. Read Full License 


\begin{abstract}
Owing to the increasing prevalence of HIV-1 CRF01_AE, it is necessary to understand the neutralization properties of CRF01_AE and to develop broadly neutralizing monoclonal antibodies (bnmAbs) that can neutralize this virus. The full-length Env gene was cloned from HIV-1 CRF01_AE-infected plasma specimens collected in China and used to establish pseudoviruses. Neutralization phenotypes of the pseudoviruses were characterized with bnmAbs. The neutralizing activities of 11 bnmAbs VRC01, VRC03, IgG1b12 and 3BNC117 (targeting the CD4 binding site); PG9 (targeting the V1V2 region); 2G12 (targeting the high mannose patch), PGT135 and 10-1074 (targeting the V3 glycans); 2F5, 4E10 and 10E8 (targeting the membrane proximal external region), against 36 pseudoviruses were analyzed, demonstrating varying efficacies. In general, VRC01, 10E8 and 3BNC117 showed strong neutralizing activity, neutralizing more than $75 \%$ of the pseudoviruses; followed by PG9 and 4E10, showing moderate neutralizing activity with neutralization of $50 \%-60 \%$ of the pseudoviruses; whereas the efficacies of the remaining bnmAbs were poor, neutralizing less than $15 \%$ of pseudoviruses tested. Env variants of CRF01_AE from one infection also showed significant differences in resistance to neutralization. These characterized HIV-1 CRF01_AE pseudoviruses could be used for neutralization studies and evaluation of vaccines or anti-HIV-1 products in China.
\end{abstract}

\title{
Introduction
}

Neutralizing antibodies (nAbs) play a critical protective role in viral diseases such as hepatitis, influenza and the novel coronavirus disease 2019 [1-3]. At a time when there is no effective vaccine to protect against human immunodeficiency virus (HIV)-1 infection [4], the protective role of nAbs has been reported [5]. These antibodies can be used for the evaluation of vaccine candidates and analysis of HIV-1 epitopes. A three-tier method for the evaluation of vaccine-induced nAbs using pseudoviruses was previously proposed [6], which is safer and more suitable compared with the use of traditional live viruses. This approach also allows for targeted mutation of amino acids and can be used for high-throughput immunogen screening [7].

To analyze each subtype systematically, researchers believe that characteristic pseudovirus panels should be developed for HIV-1 group $\mathrm{M}$ variant [7]. The CRF01_AE variant is mainly sexually transmitted and has gradually become the most prevalent subtype since its discovery in China in the 1990s [8]. As of 2020, CRF01_AE variant infections account for $37.6 \%$ of total HIV-1 infections in China[9], and the number of infections is also increasing in South and Southeast Asia. To evaluate a vaccine against the HIV-1 CRF01_AE variant, the pseudovirus library established by the National Institutes for Food and Drug Control includes some CRF01_AE pseudoviruses [10], and the pseudoviruses constructed in this study can also be included to enrich the previous pseudovirus library.

\section{Methods}

\section{Plasma samples}

The Chinese Center for Disease Control and Prevention collected plasma samples from HIV-1 subtype-infected patients from 2018 to 2019, which were subtyped according to the amplified Pol. We selected all 13 CRF01_AE plasma and successfully cloned the full length Env genes from the isolated viruses and used them to construct 36 pseudoviruses (Table S1). All samples were subtyped according to the amplified Env, and the pseudovirus samples were named according to the collection region and serial number.

\section{Cells, plasmids, and broadly neutralizing monoclonal antibodies}

HEK293T and TZM cells were obtained from the National Institutes for Food and Drug Control, Beijing, China and cultured in Dulbecco's modified Eagle's medium supplemented with $10 \%$ fetal bovine serum. Env-removed backbone plasmid pSG3 ${ }^{\Delta e n v}[23-$ 24] was obtained from the National Institutes for Food and Drug Control. Human derived broadly neutralizing monoclonal antibodies (bnmAbs) VRC01 [25], 3BNC117 [26], PGT135 [27], 10-1074 [28], 2F5 [29-30], 4E10 [31] and 10E8 [32] were provided by Tsinghua University, Beijing, China; VRC03 [25], IgG1b12 [33-36], PG9 [37] and 2 G12 [38] were obtained from the National Institutes for Food and Drug Control.

\section{Amplification, cloning and phylogenetic analysis of HIV Env}


Viral RNA was extracted from $140 \mu \mathrm{L}$ of plasma using a QIAamp viral RNA mini kit (Qiagen, Hilden, Germany). cDNA was reverse transcribed from total viral RNA using the PrimeScriptTM III first strand cDNA synthesis kit (Takara,kyoto, Japan). Primers were designed (Table S2), and the viral Env DNA fragments were amplified by nested polymerase chain reaction (PCR) using the following conditions. First round PCR amplification: pre-denaturation at $94^{\circ} \mathrm{C}$ for 4 min; 30 cycles of denaturation at $94^{\circ} \mathrm{C}$ for 15 seconds, annealing at $60^{\circ} \mathrm{C}$ for 30 seconds, extension at $68^{\circ} \mathrm{C}$ for $4 \mathrm{~min}$; extension at $68^{\circ} \mathrm{C}$ for $10 \mathrm{~min}$. Second round of PCR amplification: pre-denaturation at $94^{\circ} \mathrm{C}$ for $4 \mathrm{~min} ; 30$ cycles of denaturation at $94^{\circ} \mathrm{C}$ for 15 seconds, annealing at $60^{\circ} \mathrm{C}$ for 30 seconds, extension at $68^{\circ} \mathrm{C}$ for $3 \mathrm{~min}$; extension at $68^{\circ} \mathrm{C}$ for $10 \mathrm{~min}$. PCR products were inserted into pcDNA3.1(+) using In-Fusion enzyme (Takara) in accordance with the manufacturer's instructions.

Fifteen positively transformed DH5a colonies from each sample were selected and sequenced by the SinoGenoMax Co., Ltd/China Human Genome Center (Beijing, China) by primer walking to acquire the full-length sequence of the inserted fragment between the T7 and BGH primers of the vector. A two-parameter model with 1000 bootstraps in Mega 6.0 was used to construct a neighborjoining tree, and site-specific analysis was performed using Bioedit.

\section{Pseudovirus preparation, titration, and neutralization assay}

Plasmids were transfected into HEK293T cells using Lipofectamine 3000 reagent (Invitrogen, Carlsbad, CA). Pseudoviruses were harvested and titrated after 2 days, filtered $\left(0.45-\mathrm{mm}\right.$ pore size), and stored at $-80^{\circ} \mathrm{C}$ in $1 \mathrm{~mL}$ aliquots until use.

Neutralizing ability was measured by the decrease in Luc reporter gene expression after a single round of virus infection of TZM-BL cells. Briefly, $200 \mathrm{TCID}_{50}$ of virus was incubated with various dilutions of test mAb samples (14 dilutions in a 2-fold stepwise manner) in duplicate for 1 hour at $37^{\circ} \mathrm{C}$ in a total volume of $150 \mathrm{~mL}$ of growth medium in 96-well flat-bottom culture plates (Corning-Costar, Tokyo, Japan), together with control wells without mAbs in quadruplicate. Freshly trypsinized cells (10,000 cells in $100 \mathrm{~mL}$ of growth medium containing $37.5 \mathrm{mg} / \mathrm{mL}$ of 2-diethylaminoethyl [DEAE]-dextran) were added to each well. After 2 days of incubation, the luminescence was measured. These assays were performed as described previously [39].

\section{Statistical analysis}

A phylogenetic tree was mapped using Mega 6.0 to understand the phylogeny of the new AE strains, and S-curve testing was performed using GraphPad Prism 8.0 to assess the neutralization titer between HIV-1-positive plasma samples and mAbs. Heat maps of neutralization were established using the online heatmap tool of HIV databases (https://www.hiv.lanl.gov/content/sequence/HEATMAP/heatmap_mainpage.html). The shades of color in the map represent the strength of the response, with darker and lighter colors representing stronger and weaker responses, respectively. The ID 50 values were converted to base 10 logs before plotting.

\section{Results}

\section{Cloning the env gene and pseudovirus preparation}

Env DNA fragments were successfully amplified from 13 HIV-positive samples (Table S1). The lengths of all amplification products were as expected. The positive clones were identified and co-transfected with the backbone plasmid pSG3 ${ }^{\Delta \text { env }}$ into $293 T$ cells. Clones that could form pseudoviruses with appropriate titers were selected from each sample for further analysis. Finally, 36 pseudoviruses(Table S1) were obtained with titers ranging from 4000 to 150,000 median tissue culture infectious dose per $\mathrm{mL}$. Phylogenetic tree analysis of full-length Env showed that all 36 pseudoviruses belonged to the CRF01_AE subtype (Fig. 1).

\section{Differences in neutralization of AE variants among bnmAbs}

According to the literature [11], the 11 bnmAbs investigated were classified into five categories based on recognition sites, targeting CD4 binding sites (CD4bs), the membrane proximal extracellular region (MPER), the apical V1V2 region, the high mannose glycan on gp120, and the glycan-V3 region. The tested pseudoviruses were found to be more susceptible to two bnmAbs targeting CD4bs [VRC01(36/36) and 3BNC117(27/36)] and those targeting MPER [4E10(21/36) and 10E8(28/36)] compared with antibodies targeting other sites. The bnmAb PG9(19/36), which recognizes the apical region of the Env trimer, showed moderate neutralizing activity for the tested pseudoviruses, while a few pseudoviruses could be neutralized by the $2 \mathrm{G} 12(2 / 46)$ bnmAb, recognizing high 
mannose glycans on gp120. bnmAbs PGT135(0/46) which recognizing the glycan-V3 region was the least effective at neutralizing the pseudoviruses used in this study. Of the tested bnmAbs, VRC01 demonstrated the strongest efficacy, neutralizing $100 \%$ of the pseudoviruses tested, followed by $10 \mathrm{E} 8$ and 3BNC117, which neutralized $78 \%$ and $75 \%$ of the pseudoviruses, respectively. Both 4E10 and PG9 showed moderate neutralization rates, neutralizing $58 \%$ and $53 \%$ of the pseudoviruses, respectively; the remaining bnmAbs had poor neutralization rates for pseudoviruses at less than $15 \%$, and PGT135 failed to neutralize the tested pseudoviruses (Table 1). A heat map of the neutralization effect of the bnmAbs on different CRF01_AE-infected patient sera which these pseudoviruses were constructed from (Fig. 2A) was constructed to compare bnmAb neutralization efficiencies, and the overall neutralization abilities of the bnmAbs for all pseudoviruses in this study were represented by the geometric mean $\mathrm{IC}_{50}$, with smaller $\mathrm{IC}_{50}$ indicating stronger neutralization ability (Fig. 2B). The results showed that VRC01, 10E8 and 3BNC117 demonstrated most effective neutralization of CRF01_AE variants circulating in recent years, compared with other bnmAbs. 
Table 1

Neutralization susceptibility determined with 11 bnAbs

\begin{tabular}{|c|c|c|c|c|c|c|c|c|c|c|c|}
\hline \multirow[t]{3}{*}{ Clone } & \multicolumn{11}{|c|}{$\mathrm{IC}_{50}(\mathrm{ug} / \mathrm{mL})$} \\
\hline & \multicolumn{4}{|c|}{ gp120 CD4 binding site } & \multicolumn{3}{|c|}{ gp41 MPER } & \multirow{2}{*}{$\begin{array}{l}\text { V1V2 } \\
\text { loop } \\
\text { PG9 }\end{array}$} & \multirow{2}{*}{$\begin{array}{l}\begin{array}{l}\text { High } \\
\text { mannose }\end{array} \\
2 \mathrm{G} 12\end{array}$} & \multicolumn{2}{|c|}{ V3 glycan } \\
\hline & VRC01 & 3BNC117 & VRCO3 & IgG1b12 & 10E8 & 4E10 & $2 \mathrm{~F} 5$ & & & $\begin{array}{l}10- \\
1074\end{array}$ & PGT135 \\
\hline SZ18S0004.3 & 0.58 & 0.23 & $>10$ & $>10$ & $>10$ & 0.04 & 0.2 & $>10$ & 2.39 & 1.33 & $>10$ \\
\hline FJ18S0081.5 & 2.77 & 0.23 & 3.23 & 1.09 & 0.14 & $>10$ & $>10$ & 6.57 & $>10$ & 1.47 & $>10$ \\
\hline GD18S0125.2 & 3.45 & 0.05 & $>10$ & $>10$ & 0.06 & 0.07 & 1.09 & 1.71 & $>10$ & $>10$ & $>10$ \\
\hline 2019GX001.2 & 1.73 & $>10$ & $>10$ & $>10$ & 3.09 & $>10$ & $>10$ & $>10$ & $>10$ & $>10$ & $>10$ \\
\hline 2019GX001.3 & 1.47 & 1.85 & $>10$ & $>10$ & $>10$ & 0.7 & $>10$ & $>10$ & $>10$ & $>10$ & $>10$ \\
\hline 2019GX001.6 & 1.73 & 3.05 & $>10$ & $>10$ & 7.69 & 2.35 & $>10$ & $>10$ & $>10$ & $>10$ & $>10$ \\
\hline 2019GX001.12 & 2.38 & 0.71 & $>10$ & $>10$ & 1.21 & 1.23 & $>10$ & 3.74 & $>10$ & $>10$ & $>10$ \\
\hline 2019GX001.14 & 1.98 & 2.03 & $>10$ & $>10$ & 0.1 & $>10$ & 0.49 & 4.99 & $>10$ & $>10$ & $>10$ \\
\hline 2019GX001.15 & 2.57 & $>10$ & $>10$ & $>10$ & 1.36 & $>10$ & $>10$ & $>10$ & $>10$ & $>10$ & $>10$ \\
\hline 2019GX001.16 & 1.23 & 4.06 & $>10$ & $>10$ & 3.09 & 3.25 & $>10$ & $>10$ & $>10$ & $>10$ & $>10$ \\
\hline 2019GX001.17 & 3.06 & 0.78 & $>10$ & $>10$ & $>10$ & 0.62 & $>10$ & $>10$ & $>10$ & $>10$ & $>10$ \\
\hline 2019GX001.27 & 3.65 & 1.26 & $>10$ & $>10$ & 2.36 & 2.99 & $>10$ & 1.35 & $>10$ & $>10$ & $>10$ \\
\hline 2019GX001.44 & 1.98 & 2.31 & $>10$ & $>10$ & $>10$ & 2.35 & $>10$ & $>10$ & $>10$ & $>10$ & $>10$ \\
\hline 2019GX001.56 & 4.87 & 2.27 & $>10$ & $>10$ & 0.42 & 2.99 & $>10$ & $>10$ & $>10$ & $>10$ & $>10$ \\
\hline 2019GX001.58 & 0.52 & 0.26 & $>10$ & $>10$ & 0.92 & $>10$ & $>10$ & $>10$ & $>10$ & $>10$ & $>10$ \\
\hline 2019GX001.59 & 2.96 & 0.51 & $>10$ & $>10$ & 0.06 & 5.16 & 1.62 & 3.35 & $>10$ & $>10$ & $>10$ \\
\hline 2019GX001.60 & 2.59 & 2.23 & $>10$ & $>10$ & $>10$ & 3.2 & $>10$ & $>10$ & $>10$ & $>10$ & $>10$ \\
\hline 2019GX001.63 & 4.87 & 1.26 & $>10$ & $>10$ & 0.14 & 0.35 & 2.64 & $>10$ & $>10$ & $>10$ & $>10$ \\
\hline 2019GX001.64 & 0.52 & 2.31 & $>10$ & $>10$ & 1.2 & 5.08 & $>10$ & $>10$ & $>10$ & $>10$ & $>10$ \\
\hline 2019GX001.72 & 2.96 & 2.27 & $>10$ & $>10$ & 0.5 & 0.84 & $>10$ & $>10$ & $>10$ & $>10$ & $>10$ \\
\hline $2019 \mathrm{GX003.14}$ & 6.92 & 0.69 & $>10$ & $>10$ & 0.73 & $>10$ & $>10$ & 0.41 & $>10$ & $>10$ & $>10$ \\
\hline 2019GX014.3 & 1.02 & $>10$ & $>10$ & $>10$ & 0.71 & 6.34 & $>10$ & $>10$ & $>10$ & $>10$ & $>10$ \\
\hline 2019GX011.3 & 0.18 & 0.18 & $>10$ & $>10$ & 0.37 & $>10$ & $>10$ & 0.48 & $>10$ & $>10$ & $>10$ \\
\hline $2019 G \times 030.3$ & 1.72 & $>10$ & $>10$ & $>10$ & 0.79 & $>10$ & $>10$ & 2.1 & $>10$ & $>10$ & $>10$ \\
\hline $2019 G X 030.4$ & 1.57 & $>10$ & $>10$ & $>10$ & 0.36 & 3.68 & $>10$ & 2.21 & $>10$ & 9 & $>10$ \\
\hline 2019GX030.8 & 2.3 & $>10$ & $>10$ & $>10$ & 0.65 & 1.06 & $>10$ & 5.31 & 1.36 & $>10$ & $>10$ \\
\hline 2019GX035.7 & 0.48 & 3.68 & $>10$ & $>10$ & $>10$ & $>10$ & 0.66 & 0.09 & $>10$ & $>10$ & $>10$ \\
\hline 2019GX037.4 & 0.35 & 1.65 & 1.35 & $>10$ & 3.21 & 4.85 & $>10$ & 0.46 & $>10$ & $>10$ & $>10$ \\
\hline 2019GX037.5 & 0.35 & 2.36 & 2.48 & $>10$ & 1.65 & 5.35 & $>10$ & 0.09 & $>10$ & $>10$ & $>10$ \\
\hline 2019GX037.6 & 0.35 & 4.06 & $>10$ & $>10$ & 3.6 & $>10$ & $>10$ & 0.12 & $>10$ & $>10$ & $>10$ \\
\hline 2019GX037.9 & 0.90 & 0.24 & $>10$ & $>10$ & 3.11 & $>10$ & $>10$ & 0.17 & $>10$ & $>10$ & $>10$ \\
\hline
\end{tabular}




\begin{tabular}{|c|c|c|c|c|c|c|c|c|c|c|c|}
\hline \multirow[t]{3}{*}{ Clone } & \multicolumn{11}{|c|}{$\mathrm{IC}_{50}(\mathrm{ug} / \mathrm{mL})$} \\
\hline & \multicolumn{4}{|c|}{ gp120 CD4 binding site } & \multicolumn{3}{|c|}{ gp41 MPER } & \multirow{2}{*}{$\begin{array}{l}\begin{array}{l}\text { V1V2 } \\
\text { loop }\end{array} \\
\text { PG9 }\end{array}$} & \multirow{2}{*}{$\begin{array}{l}\begin{array}{l}\text { High } \\
\text { mannose }\end{array} \\
2 \mathrm{G} 12\end{array}$} & \multicolumn{2}{|c|}{ V3 glycan } \\
\hline & VRC01 & 3BNC117 & VRC03 & $\lg G 1 b 12$ & 10E8 & 4E10 & $2 \mathrm{~F} 5$ & & & $\begin{array}{l}10- \\
1074\end{array}$ & PGT135 \\
\hline 2019GX037.10 & 0.32 & 0.69 & $>10$ & $>10$ & 2.02 & $>10$ & $>10$ & 0.25 & $>10$ & $>10$ & $>10$ \\
\hline $2019 G \times 044.2$ & 0.83 & $>10$ & 0.76 & $>10$ & $>10$ & $>10$ & $>10$ & 0.48 & $>10$ & 1.23 & $>10$ \\
\hline 2019GX044.3 & 0.83 & $>10$ & $>10$ & $>10$ & $>10$ & $>10$ & $>10$ & 0.69 & $>10$ & 0.16 & $>10$ \\
\hline 2019GX050.1 & 0.44 & 0.29 & $>10$ & $>10$ & 0.24 & 5.36 & $>10$ & $>10$ & $>10$ & $>10$ & $>10$ \\
\hline $2019 G \times 050.9$ & 1.14 & 0.36 & $>10$ & $>10$ & 0.65 & $>10$ & $>10$ & $>10$ & $>10$ & $>10$ & $>10$ \\
\hline 2019GX057.8 & 0.74 & $>10$ & $>10$ & $>10$ & 1.23 & 1.03 & $>10$ & $>10$ & $>10$ & $>10$ & $>10$ \\
\hline
\end{tabular}

\section{Effect of Env variants on neutralizing activity}

Seventeen Env clones were amplified from 2019GX001, and the resulting pseudoviruses had different neutralization characteristics when exposed to bnmAbs, showing escape from single antibodies (Fig. 3A). Of the 2019GX001 variants, 2019GX001.59 showed highest susceptibility to neutralization by six bnAbs, comprising VRC01, 10E8, 3BNC117, 4E10, 2 F5 and PG9. Four pseudoviruses could be neutralized by five bnmAbs, comprising 2019GX001.12, 2019GX001.14, 2019GX001.27 and 2019GX001.63, four pseudoviruses could be neutralized by four bnmAbs, including 2019GX001.16, 2019GX00156, 2019GX001.64 and 2019GX001.72, and six pseudoviruses could be neutralized by three bnmAbs, including 2019GX001.3, 2019GX001.6,2019GX001.17, 2019GX001.44, 2019GX001.58 and 2019GX001.60. Two pseudoviruses-2019GX001.2 and 2019GX001.15-were neutralized by VRC01 and 10E8 only, whereas all other bnmAbs were ineffective against these pseudoviruses. Five Env clones were obtained from $2019 \mathrm{GX037}$ and three of the five showed resistance to VRC03 and 4E10 and these viruses were susceptible to the remaining bnmAbs, including VRC01, 10E8 and 3BNC117 (Fig. 3B).

\section{Discussion}

This study constructed 36 CRF01_AE pseudoviruses which contain full-length env gene of CRF01_AE subtype and these pseudoviruses could be used for neutralization studies and evaluation of vaccines or anti-HIV-1 products in China. The sera of infected or vaccinated individuals were polyclonal antibodies, which can not clearly indicate which epitopes are mainly neutralized by antibodies. Therefore, the use of bnmAbs that can recognize different epitopes can reflect the neutralization effect of pseudovirus on polyclonal antibodies (the sera of vaccinated persons). So we analyzed the the neutralizing activities of 11 bnmAbs against these pseudoviruses. We identified several potential sites that may affect the neutralization activity of bnmAbs in the process.

According to the results of this study, it was hypothesized that bnmAbs recognizing CD4bs(VRC01 and 3BNC117) and MPER(4E10 and 10E8) would have the best neutralizing activity against CRF01_AE pseudoviruses. Although the antibodies were not the same as those we used, Wang's research showed that bnmAbs target MPER have the best neutralizing, too[12]. Earlier, Chenine et al. reported that bnmAbs recognizing CD4bs and MPER also showed good neutralizing activity against CRF01_AE pseudoviruses from Thailand[13], Japan and Vietnam[14]. Ju et al. first isolated HIV-1 nAbs named F6 from a CRF01_AE-infected donor, it may be more suitable for neutralizing CRF01_AE pseudovirus[15]. We are trying to get F6 now.

VRC01 and VRC03 are two of the bnmAbs which target the CD4 binding site, they are closely related. However, in this study, VRC03 doesn't neutralize most of pseudoviruses, although VRC01 did all. Is the low potency of VRC03 CRF01_AE-specific? Through Xie's paper, we found that she same situation is true for subtype B virus. As we know that most of the epitopes recognized by vrc03 can also be recognized by VRC01, and VRC01 is able to recognize more epitopes than VRC03. This may be the reason for this results. 
The characterization of 36 CRF01_AE pseudovirus variants with 11 bnmAbs identified a number of sites that may affect the neutralization activity of bnmAbs. Antibody 3BNC117 recognizes a discontinuous epitope on gp120, and its neutralization activity is affected by changes at several sites (including P124, T198, V275, A281, R308, S365, Q428, G459, N462, and G471), of which amino acid changes at the G459 site makes the virus more susceptible to neutralization by 3BNC117[16]. Seven of the 36 pseudoviruses used in this study (2019GX001.12, 2019GX001.14, 2019GX001.15, 2019GX001.44, 2019GX001.56, 2019GX001.58 and 2019GX001.59) showed amino acid changes at G459D, while one (2019GX001.15) was resistant to 3BNC117, suggesting that this site may not be the most critical site for neutralizing activity. One pseudovirus, 2019GX014.3, demonstrated a P124Y change and was resistant to 3BNC117, suggesting that this site may have some effect on 3BNC117 neutralizing activity. Pseudoviruses 2019GX001.2, 2019GX001.3, 2019GX001.6, 2019GX001.12, 2019GX001.56 and 2019GX001.59 were obtained from a single patient, and only $2019 \mathrm{GX001.2}$ showed resistance to 3BNC117, with K282R as its specific amino acid change, suggesting that this locus may have an effect on susceptibility to 3BNC117.

Antibody $2 \mathrm{G} 12$ is a glycosylation-dependent bnmAb that recognizes glycosylated conformational epitopes, including N295, N332, N386, N392, N397 and N448 [17]; these epitopes have been reported to play an important role in the binding of 2 G12 to gp120 [18]. Only two of the 36 pseudoviruses used in this study could be neutralized by 2G12, and the 34 pseudoviruses showing no susceptibility to $2 \mathrm{G} 12$ had varying mutations at the above-mentioned loci, suggesting that N295 and N332 play an important role in binding. The two pseudoviruses SZ18S0004.3 and 2019GX030.8 that were highly susceptible to 2 G12 carried $339 \mathrm{~N}$ in addition to $295 \mathrm{~N}$ and $332 \mathrm{~N}$, suggesting that the $\mathrm{N} 339$ position may also be important for $2 \mathrm{G} 12$ recognition of the virus.

The core sequence of the epitope recognized by the $4 \mathrm{E} 10 \mathrm{bnmAb}$ is located at positions $671-676$ with the sequence NWFNIT, with additional important binding related sites. Previous reports have shown that three sites $-672 \mathrm{~W}, 673 \mathrm{~F}$ and $680 \mathrm{~W}-$ are critical for the neutralization activity of antibodies [19], but the relationship between amino acid mutations in the above sites and neutralization susceptibility in these 36 pseudoviruses showed no regular patterns. In contrast, four strains of 2019GX001, 2019GX001.2, 2019GX001.58, 2019GX001.14, and 2019GX001.15, demonstrated resistance to 4E10, and the simultaneous presence of amino acid changes in G691S in these four strains suggested that this locus may affect the neutralizing activity of $4 \mathrm{E} 10$ against the virus.

The epitopes recognized by $10 \mathrm{E} 8$ overlap and are more numerous than those recognized by $4 \mathrm{E} 10$, and this bnmAb recognizes NWFDITNWLWYIK at positions 671-683 [20], where 671N, 672W, 673F, 676T, 680W and 683K/R are critical for $10 \mathrm{E} 8$ binding and neutralization of the virus. It has also been reported that glycosylation at positions $231 \mathrm{E}$ and 301 could increase the neutralization efficacy of 10E8, while $693 \mathrm{~V}$ reduced neutralization by $10 \mathrm{E} 8$. Comparison of the sequences revealed that the amino acid changes and neutralizing activity of these 36 pseudoviruses at the above-mentioned loci remained inconsistent, but one pseudovirus, 2019 GX001.3 of the 2019GX001 variants, also showed resistance to 10E8, and the only unique mutation site in this variant compared with others was T90A, indicating that this site most likely affected neutralization of the virus by $10 \mathrm{E} 8$.

The presence of antibodies in an infected person can drive the virus to mutate, and the antibodies produced in an HIV- 1 infected person can stimulate the evolution of the virus that established the infection; even if the patient has not been treated with a bnmAb, it is possible that the same dominant variant in the body will develop quasi-species of escape mutations against bnmAb. For this reason, among others, researchers have tried to use a "cocktail" approach to increase the broad-spectrum and neutralizing activity in bnmAb treatment, which is already available in "3BNC117+10-1074" therapy [21]. Among the observed escapes of virus from bnmAbs in this study, the 2019GX001 and 2019GX037 strains both could be neutralized by VRC01, suggesting that the site recognized by VRC01 may be more conserved, and it may be the most suitable bnmAb for the treatment of CRF01_AE. Meanwhile, 10E8 showed the second strongest neutralizing activity. Thida et al. found that VRC01 and 10E8 also had the strongest neutralizing activity against CRF01_AE from Japan and Vietnam[14], suggesting that "VRC01 + 10E8" therapy may be promising for the treatment of CRF01_AE-infected patients.

Pseudoviruses, as a novel tool with the advantages of safety, high throughput, and the possibility of targeted amino acid mutations, are gradually being used in vaccine evaluation, and their sensitivity may exceed that of viral infection experiments [22].The 36 pseudoviruses from 13 CRF01_AE samples used in this study belong to cluster 1, cluster 2, cluster 4 and cluster 5 respectively. We found that these HIV-1 CRF01_AE pseudoviruses showed higher susceptibility to antibodies against CD4 binding sites and MPER than antibodies against V1V2 and V3 regions. Among them, three bnmAbs-VRC01, 10E8 and 3BNC117-showed

Page $7 / 13$ 
the highest neutralizing potency against CRF01_AE variants. For the cluster3, cluster6 and cluster7, we will collect them in the future. Considering the high mutagenicity of the HIV-1 virus, combination bnmAb therapy holds promise for successful treatment of HIV-1 infection.

\section{Declarations}

\section{Acknowledgments}

We thank Gillian Campbell, PhD, from Liwen Bianji (Edanz) (www.liwenbianji.cn/), for editing the English text of a draft of this manuscript.

We thank the support from Prof.Linqi Zhang of Tsinghua University for providing antibodies.

\section{Authors' contributions}

XY Zhang and WJ Huang wrote the main manuscript text and all authors prepared figures 1-3. All authors reviewed the manuscript

\section{Funding}

This work was supported by National Science and Technology Major Projects of Infectious Disease under Grand 2017ZX10201101-003-002

\section{Availability of data and materials}

All data and material are available upon request to correspondence author.

\section{Ethics approval and consent to participate}

All experimental protocols were approved by National Center for AIDS/STD Control and Prevention, China CDC.

All procedures performed in studies involving human participants were in accordance with the ethical standards of the institutional and/or national research committee and with the 1964 Helsinki declaration and its later amendments or comparable ethical standards.

Informed consent was obtained from all individual participants included in the study. All subjects are over 18 years old.

\section{Consent for publication}

Not applicable.

\section{Competing interests}

The author(s) declare no competing interests.

\section{References}

1. Cao Y, et al. Potent Neutralizing Antibodies against SARS-CoV-2 Identified by High-Throughput Single-Cell Sequencing of Convalescent Patients' B Cells. Cell. 182(1),73-84.e16 (2020).

2. Corti D, Cameroni E, Guarino B, Kallewaard NL, Zhu Q, Lanzavecchia A. Tackling influenza with broadly neutralizing antibodies. Curr Opin Virol. 24,60-69 (2017).

3. Hong B, Wen Y, Ying T. Recent Progress on Neutralizing Antibodies against Hepatitis B Virus and its Implications. Infect Disord Drug Targets. 19(3),213-223 (2019).

4. Burton DR. Advancing an HIV vaccine; advancing vaccinology. Nat Rev Immunol. 19(2),77-78 (2019).

5. Burton DR, Hangartner L. Broadly Neutralizing Antibodies to HIV and Their Role in Vaccine Design. Annu Rev Immunol. 34,635-59 (2016). 
6. Mascola JR, D'Souza P, Gilbert P, et al. Recommendations for the design and use of standard virus panels to assess neutralizing antibody responses elicited by candidate human immunodeficiency virus type 1 vaccines.J Virol. 79,1010310107 (2005).

7. Wang S, Nie J, Wang Y. Comparisons of the genetic and neutralization properties of HIV-1 subtype C and CRF07/08_BC env molecular clones isolated from infections in China. Virus Res. 155(1),137-146 (2011).

8. Feng Y, He X, Hsi JH, Li F, Li X, Wang Q, Ruan Y, Xing H, Lam TT, Pybus OG, Takebe Y, Shao Y. The rapidly expanding CRF01_AE epidemic in China is driven by multiple lineages of HIV-1 viruses introduced in the 1990s. AIDS. 27(11),1793-802 (2013).

9. HIVdatabase. Distribution of all HIV-1 sequences

10. https://

11. Nie J, Huang W, Liu Q, Wang Y. HIV-1 pseudoviruses constructed in China regulatory laboratory. Emerg Microbes Infect. 9(1),32-41 (2019).

12. HIV Molecular Immunology Database. Standardized Assessments of Neutralizing Antibodies for HIV/AIDS Vaccine Development [EB]. Available from: https://www.hiv.lanl.gov/content/nab-reference-strains/html/home.htm

13. Thida W, Kuwata T, Maeda Y, et al. The role of conventional antibodies targeting the CD4 binding site and CD4-induced epitopes in the control of HIV-1 CRF01_AE viruses. Biochem Biophys Res Commun. 508(1):46-51 (2019).

14. Wang H, Yuan T, Li T, et al. Evaluation of susceptibility of HIV-1 CRF01_AE variants to neutralization by a panel of broadly neutralizing antibodies. Arch Virol. 163(12):3303-3315 (2018).

15. Chenine AL, Merbah M, Wieczorek L, et al. Neutralization Sensitivity of a Novel HIV-1 CRF01_AE Panel of Infectious Molecular Clones. J Acquir Immune Defic Syndr. 78(3):348-355 (2018).

16. Ju B, Li D, Ren L, et al. Identification of a novel broadly HIV-1-neutralizing antibody from a CRF01_AE-infected Chinese donor. Emerg Microbes Infect. 7(1):174 (2018).

17. Morales JF, Yu B, Perez G, Mesa KA, Alexander DL, Berman PW. Fragments of the V1/V2 domain of HIV-1 glycoprotein 120 engineered for improved binding to the broadly neutralizing PG9 antibody. Mol Immunol. 77, 14-25 (2016).

18. Scanlan $\mathrm{CN}$, et al. The broadly neutralizing anti-human immunodeficiency virus type 1 antibody $2 \mathrm{G} 12$ recognizes a cluster of alpha1->2 mannose residues on the outer face of gp120 [J]. J Virol. 76 (14), 7306-7321 (2002).

19. Shang $\mathrm{H}$, et al. Genetic and neutralization susceptibility of diverse HIV-1 env clones from chronically infected patients in China [J]. J Biol Chem. 286 (16), 14531-14541 (2011).

20. Zwick MB, et al. Anti-human immunodeficiency virus type 1 (HIV-1) antibodies $2 \mathrm{~F} 5$ and $4 \mathrm{E} 10$ require surprisingly few crucial residues in the membrane-proximal external region of glycoprotein gp41 to neutralize HIV-1 [J]. J Virol. 79 (2), 12521261(2005).

21. Rujas E, et al. Structural basis for broad neutralization of HIV-1 through the molecular recognition of $10 \mathrm{E} 8$ helical epitope at the membrane interface [J]. Sci Rep. 6, 38177-38189 (2016).

22. Cohen YZ, Butler AL, Millard K, et al. Safety, pharmacokinetics, and immunogenicity of the combination of the broadly neutralizing anti-HIV-1 antibodies 3BNC117 and 10-1074 in healthy adults: A randomized, phase 1 study. PLoS One. 14(8), e0219142 (2019).

23. Fenyo EM, Heath A, Dispinseri S, et al. International network for comparison of HIV neutralization assays: the NeutNet report. PLOS ONE. 4, e4505 (2009).

24. Wei X, Decker JM, Liu H, et al. Emergence of resistant human immunodeficiency virus type 1 in patients receiving fusion inhibitor (T-20) monotherapy. Antimicrob Agents Chemother. 46,1896-1905 (2002).

25. Wei X, Decker JM, Wang S, et al. Antibody neutralization and escape by HIV - 1. Nature. 422,307-312 (2003).

26. Walensky, R.P., et al., The survival benefits of AIDS treatment in the United States. J Infect Dis, 194(1), p. 11-9(2006).

27. Shingai M, Nishimura Y, Klein F, et al. Antibody-mediated immunotherapy of macaques chronically infected with SHIV suppresses viraemia. Nature. 503(7475),277-280 (2013).

28. Pritchard LK, Spencer DI, Royle L, et al. Glycan Microheterogeneity at the PGT135 Antibody Recognition Site on HIV-1 gp120 Reveals a Molecular Mechanism for Neutralization Resistance. J Virol. 89(13),6952-6959 (2015). 
29. Caskey M, Schoofs T, Gruell H, et al. Antibody 10-1074 suppresses viremia in HIV-1-infected individuals. Nat Med. 23(2):185191 (2017).

30. Purtscher $\mathrm{M}$, et al. Restricted antigenic variability of the epitope recognized by the neutralizing gp41 antibody $2 \mathrm{~F} 5$ [J]. AIDS. 10 (6),587-593 (1996).

31. Purtscher $\mathrm{M}$, et al. A broadly neutralizing human monoclonal antibody against gp41 of human immunodeficiency virus type 1 [J]. AIDS Res Hum Retroviruses. 10 (12),1651-1658 (1994).

32. Stiegler $\mathrm{G}$, et al. A potent cross-clade neutralizing human monoclonal antibody against a novel epitope on gp41 of human immunodeficiency virus type 1 [J].AIDS Res Hum Retroviruses. 17 (18), 1757-1765 (2001).

33. Huang J, et al. Broad and potent neutralization of HIV-1 by a gp41-specific human antibody [J]. Nature. 491 (7424), 406-412 (2012).

34. Burton DR, et al. A large array of human monoclonal antibodies to type 1 human immunodeficiency virus from combinatorial libraries of asymptomatic seropositive individuals [J]. Proc Natl Acad Sci U S A. 88 (22), 10134-10137 (1991).

35. Barbas CF 3rd, et al. Recombinant human Fab fragments neutralize human type 1 immunodeficiency virus in vitro [J]. Proc Natl Acad Sci U S A. 89 (19), 9339-9343 (1992).

36. Burton DR, et al. Efficient neutralization of primary isolates of HIV-1 by a recombinant human monoclonal antibody [J]. Science. 266 (5187), 1024-1027 (1994).

37. Roben P, et al. Recognition properties of a panel of human recombinant Fab fragments to the CD4 binding site of gp120 that show differing abilities to neutralize human immunodeficiency virus type 1 [J]. J Virol. 68 (8), 4821-4828 (1994).

38. Walker LM, Phogat SK, Chan-Hui PY, et al. Broad and potent neutralizing antibodies from an African donor reveal a new HIV-1 vaccine target. Science. 326(5950), 285-289 (2009).

39. Luo XM, Lei MY, Feidi RA, et al. Dimeric 2 G12 as a potent protection against HIV-1. PLoS Pathog. 6(12), e1001225 (2010).

40. Chong H, Hong K, Zhang C, Nie J, Song A, Kong W, Wang Y. Genetic and neutralization properties of HIV-1 env clones from subtype B/BC/AE infections in China. J Acquir Immune Defic Syndr. Apr 15;47(5), 535-43 (2008).

\section{Figures}




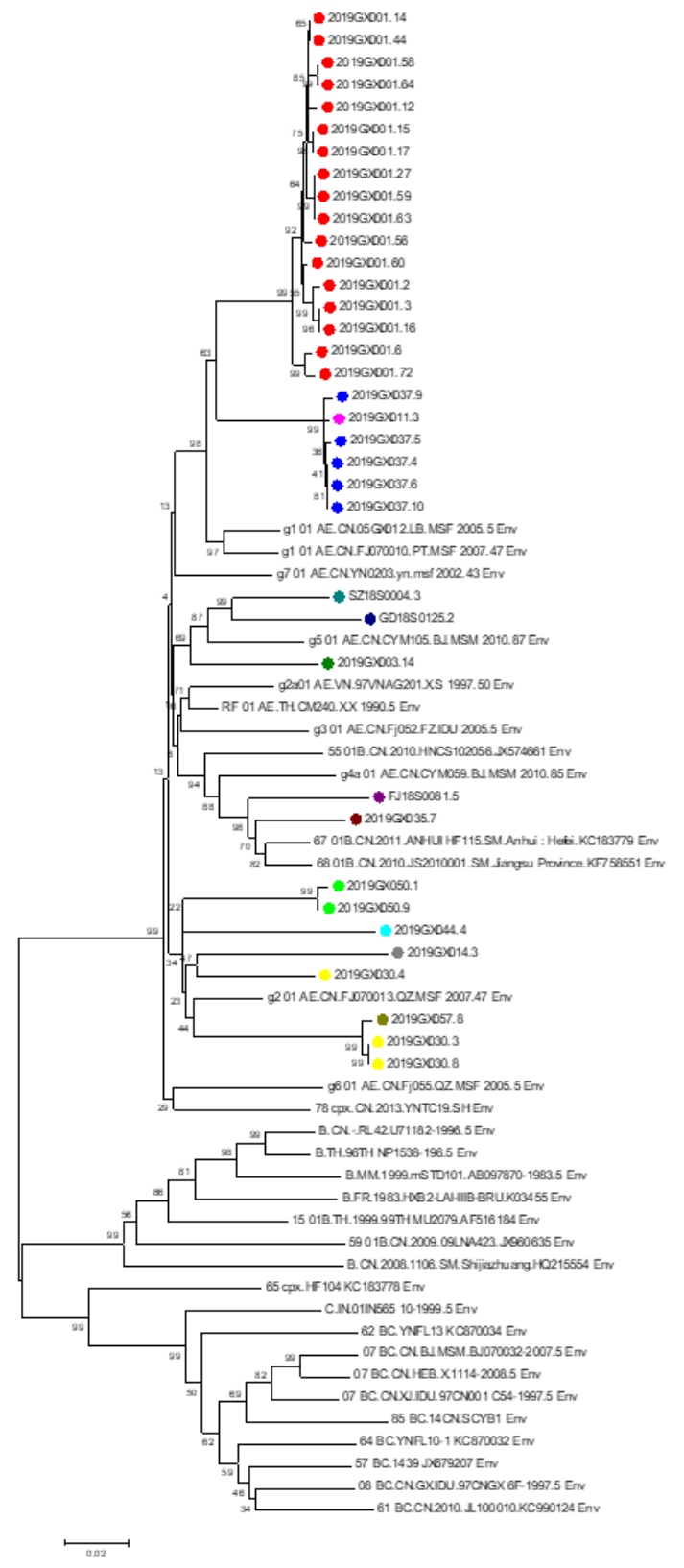

Figure 1

Phylogenetic analysis of the newly sequenced HIV-1 Env genes alongside reference strains. The newly constructed Env clones are indicated by solid circles, with circles of the same color representing the same plasma source and the rest being reference strain Env clones. Lengths of horizontal branches are drawn to scale (scale bar indicates 0.02 nucleotide substitutions per site), while vertical separations are for clarity only. Values at nodes indicate the percentage of bootstraps in which the cluster to the right was found. 
A

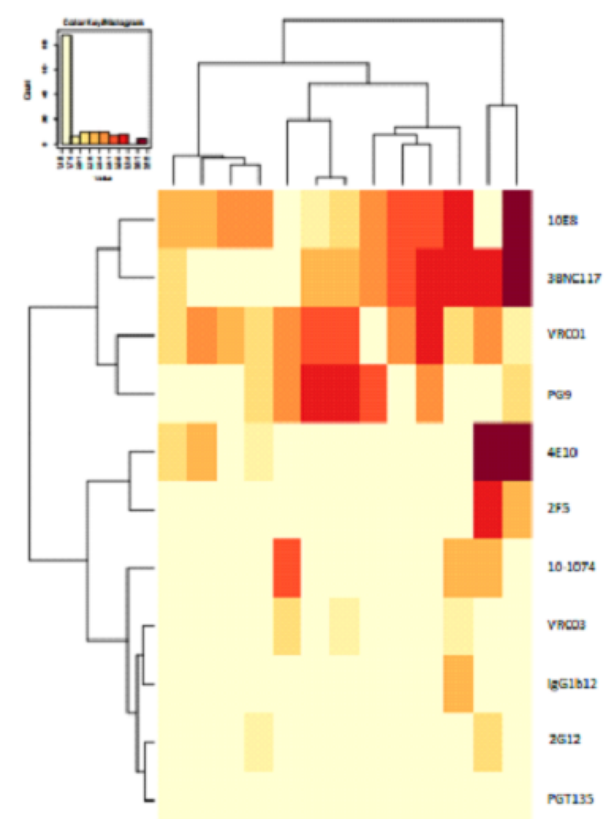

IIIIIIII

B

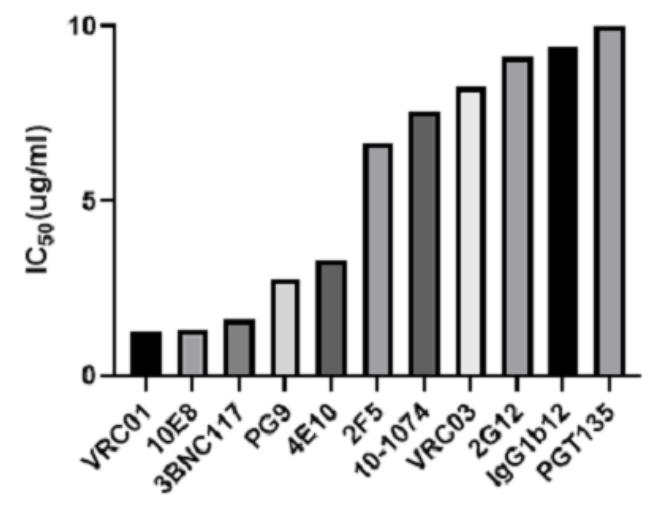

Figure 2

(A) Heat map analysis of bnmAb neutralization of pseudoviruses from different infected individuals (the geometric mean of bnmAb neutralization of pseudoviruses from the same individual was used for comparison when multiple pseudoviruses were constructed from one infected individual). The magnitude of neutralization (inhibition relative to light units) is expressed as a color, with lower values represented by less saturated lighter colors and higher neutralization values represented by more saturated darker colors. (B) Differences in neutralizing potency of bnmAbs against HIV-1 CRF01_AE pseudoviruses. Geometric means of bnmAb neutralization against 36 pseudoviruses were used for comparison; for IC $50>10 \mu \mathrm{g} / \mathrm{mL}$, the graph was calculated with IC50 of $10 \mu \mathrm{g} / \mathrm{mL}$. 
A

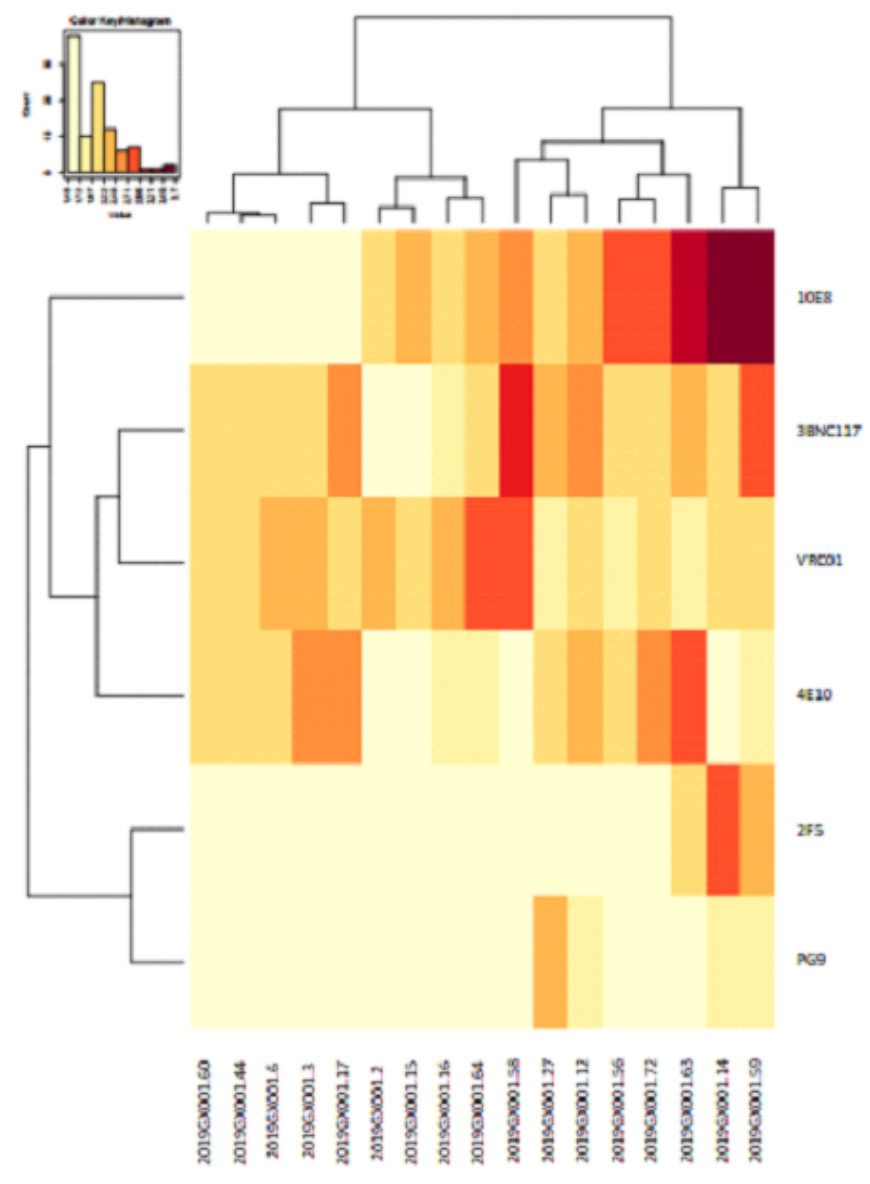

B
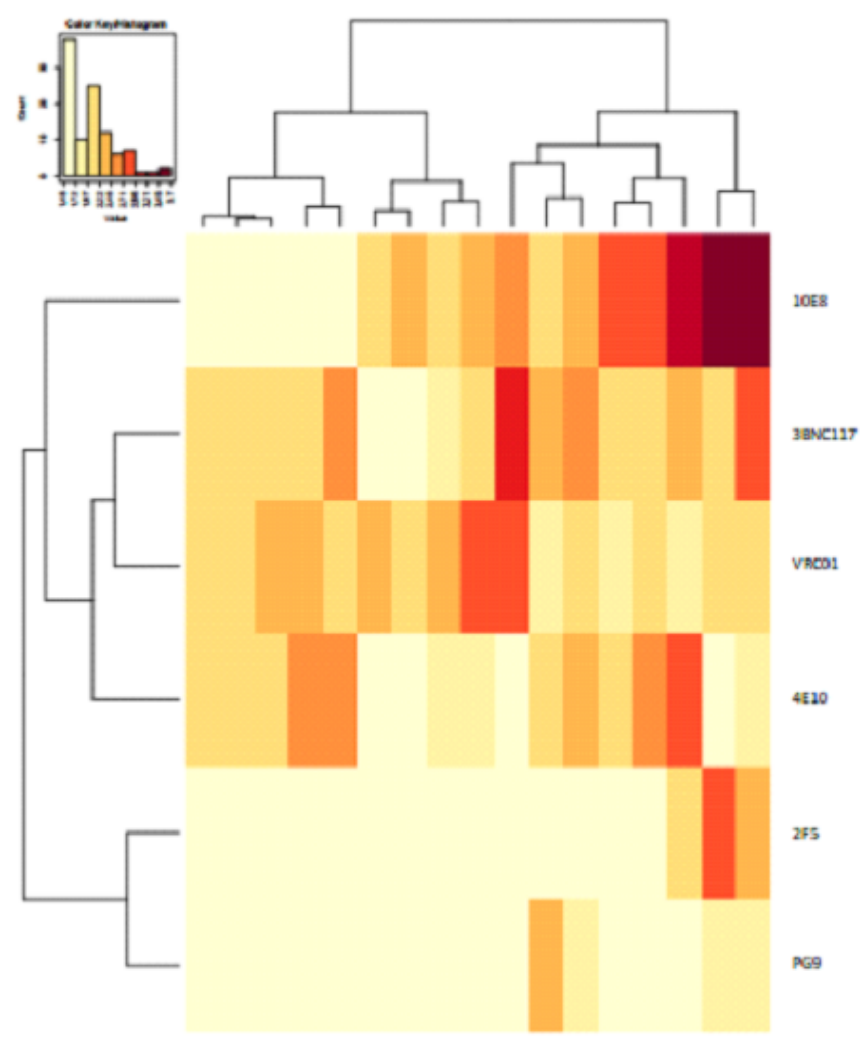

IIIIIIIIIIIIII

\section{Figure 3}

Heat map analysis of bnmAb neutralization of different pseudoviruses from two infected individuals, (A) 2019GX001 and (B) 2019GX037, with the magnitude of neutralization (inhibition relative to light units) indicated by color. Lower values are represented by less saturated light colors, higher neutralization values are represented by more saturated dark colors. bnmAbs that failed to neutralize any of the tested pseudoviruses are not shown.

\section{Supplementary Files}

This is a list of supplementary files associated with this preprint. Click to download.

- Supplementary.docx 